\title{
TRAVAUX RÉCENTS SUR LA CLASSIFICATION
}

\section{DES DERMATOPHYTES. - II (1)}

\author{
Par Maurice LANGERON
}

Au moment (1926) où parut le premier travail de L. Grigorakis, présenté à la Sorbonne comme thèse de doctorat d'Université et intitulé Recherches sur les dermatophytes, j'ai exprimé mes réserves au sujet de la classification que cet auteur avait cru devoir adopter. Soit dans ces Annales, au cours d'une Revue critique (1), soit dans le Bulletin de l'Institut Pasteur (2), dans l'analyse critique de ce mémoire, j'ai dit que " cette classification avait peu de chances d'être adoptée, car elle méconnaît trop ouvertement les règles internationales de la nomenclature scientifique ". Cette opinion n'a pas varié ; elle s'est même renforcée à la suite de la publication récente, d'une part par J. Guiart et L. Grigorakis, d'autre part par L. Grigorakis, de cette même classification légèment modifiée.

On peut se demander tout d'abord pourquoi le nom de J. Guiart se trouve en tête de cette classification, publiée dans un article intitulé : La classification botanique des champignons des teignes, paru dans le Lyon médical du $1^{\text {er }}$ avril 1928 et précédant de peu la distribution de la thèse de doctorat universitaire (médecine) de L. Grigorakis (Contribution à l'étude des teignes et de leurs parasites, Lyon, 1928). Qu'un professeur présente, comme c'est le cas pour cette thèse, dans une préface élogieuse, le travail de son assistant et élève, la chose est normale. Mais, dans la publication commune à laquelle je fais allusion, on ne voit pas exactement la part de collaboration de chacun, puisque la classification qu'elle expose n'est en somme que l'œuvre un peu modifiée de Grigorakis, publiée dans ses lignes essentielles plus de trois ans auparavant. Il en résulte que J. Guiart, sans avoir fait paraître de travaux particuliers sur la question, puisque je n'en ai trouvé aucune trace dans la bibliographie, assume la responsabilité des opinions émises par L. Grigorakis et doit prendre sa part des critiques formulées plus

(1) LANGenon (M.): - Travaux récents sur la classification des dermatophytes. Ann. de Parasitologie, IV, 1926. p. 193-198.

(2) Bull. Institut Pasteur, XXIII, 1925, p. 442, XXIV, 1926, p. 392-394, XXV, 1927, p. 1048 .

Annales de Parasitologie, T. VI, N $^{\circ} 4 .-1^{\text {er }}$ octobre 1928, p. 470-476. 
haut par notre éminent Maître, R. Sabouraud, et de celles que je vais, à mon tour, présenter, aux points de vue systématique et botanique.

L'impression qui se dégage de la lecture des mémoires de Guiart et Grigorakis est que la confusion est arrivée à son comble et qu'il semble difficile de la porter à un plus haut degré de perfection. Sous prétexte de conserver les trois anciens genres : Trichophyton, Microsporum et Achorion, ou d'y revenir, on enlève à ceux-ci leur signification primitive pour y faire entrer des types disparates, de sorte que ces genres ne correspondent plus aux types établis par leurs créateurs.

La conservation des trois anciens genres fondamentaux serait une chose excellente si l'on pouvait constituer ainsi une clef à double entrée, dans laquelle ces trois genres faciliteraient un premier triage, d'après les caractères que les dermatophytes présentent dans les lésions ; les nouveaux genres ou sous-genres permettraient ensuite de répartir les espèces d'après les caractères botaniques plus complets qu'elles présentent dans les cultures sur milieux artificiels. Tel n'est pas le résultat obtenu par Guiart et Grigorakis. Par exemple, on trouve dans le genre Microsporum nouveau style l'Epidermophyton inguinale, qui ne rappelle en rien, ni à l'état parasitaire, ni sous la forme culturale, les caractères admis jusqu'ici pour les Microsporum, ou l'ancien Achorion gallinæ, dont Altara a démontré la nature trichophytique. De même, on voit rapprochés dans le genre Achorion nouveau style des champignons aussi dissemblables que l'Achorion (Grubyella) schönleini et l'Endodermophyton concentricum. En un mot, les termes Microsporum, Trichophyton, Achorion, signifiaient autrefois quelque chose de précis, sur quoi tout le monde était d'accord. G. et G. leur enlèvent cette signification pour leur en donner une nouvelle, toute différente, si bien que ce sont en réalité de nouveaux types génériques sous un nom ancien. D'où la confusion extrême dont j'ai parlé et qui fait tomber ces anciens noms, prenant un sens nouveau, sous le coup du $\$ 4$ de l'article 51 du Code de nomenclature. On en revient donc à la nécessité de créer des noms nouveaux.

Or, ces noms existent, ce sont ceux qui ont été proposés en 1923 par Ota et Langeron, car, plus que jamais, les deux classifications coïncident. Sur les 15 espèces formant le genre Microsporum G. et G. nouveau style, 14 sont des Sabouraudites ; une seule, le Microsyorum inguinale, est précisément cet Epidermophyton, si particulier, si bien caractérisé, qu'il mérite à lui seul les honneurs d'un genre. Passons au genre Trichophyton nouveau style: sur 11 espèces, il y a 9 Trichophyton au sens d'Ota et Langeron, et 2 Sabouraudites. Les subdivisions du genre Achorion nouveau style 
sont nos trois genres Grubyella, Bodinia et Endodermophyton; ce dernier est conservé tel quel, tandis que quelques Grubyella passent chez les Bodinia, sans que ce déménagement soit motivé par des raisons impérieuses.

Guiart et Grigorakis nous ont fait l'honneur d'une longue critique, s'efforçant de démontrer que leur classification diffère beaucoup de la nòtre. Ils attaquent surtout notre genre Sabouraudites. Il faut cependant que ce groupement corresponde à quelque chose de réel, puisque nous le retrouvons à peu près intact, quoique sous un autre nom, dans leur classification. L'aménagement de ses sousgenres présente, on en conviendra, une importance bien moins grande ; c'est une question de dosage des aleuries et des fuseaux. Il faudrait discuter ici pied à pied pour chaque espèce, ce que j'épargnerai au lecteur ; G. et G. nous font beaucoup d'honneur en y consacrant 14 pages. D'ailleurs, la signification des sous-genres est à peu près nulle. Le sous-genre est un mauvais groupement : ou bien les espèces qu'on y range présentent un ensemble de caractères tranchés, et alors il faut les réunir dans un genre, pour leur donner une individualité systématique; ou bien il s'agit de cataloguer de trop nombreuses espèces affines et alors les catégories ainsi établies seront toujours peu nettes et sujettes à variations suivant l'opinion de chacun. La nomenclature étant binaire, on pourrait même dire que le sous-genre n'a pas de raison d'être.

La confusion vient aussi des principes dont G. et G. sont partis : examinons-les rapidement :

1. - Le premier est que des champignons très différents peuvent produire le même syndrome clinique et qu'inversement une même espèce peut causer plusieurs types de lésions. Cette vérité était déjà connue ; on sait depuis longtemps que chaque tissu réagit pour son compte et suivant un type défini. La gomme et le tubercule peuvent être produits par des parasites très divers et mème par des corps inertes. L'épiderme desquame, suinte, se soulève en vésicules ou pustules sous les influences les plus variées, etc., etc. Tout cela n'a rien à voir avec une classification botanique; et même à ce point de vue, des ressemblances très étroites peuvent se produire, par suite du phénomène d'épharmonie, si bien mis en lumière par Vesque, et qui se manifeste dans la généralité du règne végétal.

L'épharmonie est l'état de la plante adaptée. Cette adaptation au milieu, conditionnée par les facteurs écologiques, porte surtout sur le système végétatif et beaucoup moins sur l'appareil reproducteur. Ce dernier est plutôt sous la dépendance des facteurs d'hérédité, dont-l'influence fait persister des caractères ancestraux, pouvant être en désaccord avec le milieu. Ce sont ces caractères héréditaires 
de l'appareil reproducteur qui ont une importance systématique. Les autres peuvent être très trompeurs, en vertu de la convergence épharmonique qui fait apparaître des caractères végétatifs identiques chez des végétaux très éloignés les uns des autres. Un des exemples les plus typiques de convergence épharmonique est le facies cactoïde, réalisé à la fois par les cactées et par des euphorbiacées et des asclépiadées.

C'est la convergence épharmonique qui donne à tous les champignons parasites de la peau et des poils une grande similitude de caractères végétatifs. Mais cette analogie, qui se manifeste à l'état parasitaire, ne subsiste pas toujours dans les cultures, c'est-à-dire à l'état saprophytique. C'est ce qui permet de séparer les Aleurisma, Malassezia, Trichosporum, etc., des dermatophytes proprement dits ou champignons des teignes. Chez ces derniers, c'est la convergence épharmonique qui produit les formes végétatives trichophytique, microsporique et favique, types des trois anciens genres fondamentaux, basés sur les caractères du champignon à l'état parasitaire. Néanmoins, les dermatophytes forment un groupe bien homogène, parce qu'en dehors des caractères épharmoniques, ils présentent encore un ensemble de caractères morphologiques et biologiques communs : aleuries, fuseaux, polymorphisme mycélien, pléomorphisme.

2. - Le deuxième principe repose sur une série d'affirmations erronées. G. et G. écrivent que les dermatophytes se pléomorphisent toujours, que ce pléomorphisme est une forme spéciale du polymorphisme et qu'il se manifeste par une dégradation. Il y a là d'abord une erreur de fait que Sabouraud a relevée plus haut. Je n'insisterai donc pas sur ce point, chacun sachant que, gràce au milieu de conservation de Sabouraud, il est possible d'inhiber le pléomorphisme et d'en préserver les collections de cultures de dermatophytes. Mais, à mon sens, G. et G. donnent du terme pléomorphisme une interprétation inexacte. Pour eux, le pléomorphisme est caractérisé par une dégradation constante, plus ou moins lente à apparaître, mais fatale, quelles que soient les conditions. Ce n'est pas toujours vrai, comme nous venons de le voir, mais enfin prenons le cas où le champignon se pléomorphise. Assistons-nous réellement à une dégradation? Et d'abord, qu'entend-on par dégradation? Si c'est une simplification morphologique et biologique, celle-ci ne peut guère être poussée plus loin que pendant la vie parasitaire du champignon dans le poil ou dans l'épiderme. Pourtant il ne s'agit pas là de pléomorphisme, bien que les organes reprodueteurs n'apparaissent jamais et que le champignon soit réduit à des filaments et à des globules ou spores mycéliennes. Le pléomorphisme est-il une " forme spéciale du polymorphisme ", comme le veulent G. et G. ? 
Le dire, c'est méconnaître son caractère essentiel, ear, au sens où Sabouraud applique ce terme à l'évolution des dermatophytes, il s'agit d'une véritable mutation, c'est-à-dire de l'apparition de caractères nouveaux et, fait unique, d'une mutation stable. En effet, d'après les expériences de Sabouraud, une culture pléomorphisée ne revient jamais à la forme primitive, ni par ensemencement sur milieux appropriés, ni même par inoculation à l'animal et rétro. culture. Sabouraud a donc découvert un fait sans analogue et d'une portée considérable.

Mais tout cela n'a rien à voir avec la classification des dermatophytes, puisque celle-ci doit être établie, comme Grigorakis le répète avec insistance, au moyen de cultures-mères non pléomorphisées. Or, Sabouraud a démontré expérimentalement que celles-ci peuvent être indéfiniment reproduites identiques à elles-mêmes. Le deuxième principe de la classification de G. et G. repose done sur une erreur de fait et se trouve de plus inutile.

3. - Un troisième principe est formulé moins nettement, mais ressort de la critique faite par G. et G. des autres classifications. Ces auteurs refusent toute valeur systématique aux formations mycéliennes : organes pectinés, organes nodulaires, tortillons spiralés, vrilles, etc. Ils estiment, d'autre part, que les divers corps propagateurs qu'on observe chez les dermatophytes " résultent les uns des autres ", affirmation à l'appui de laquelle aucune preuve n'est apportée.

Evidemment, dans toute classification naturelle, il doit $\mathrm{y}$ avoir subordination des caractères: ceux qui sont tirés de l'appareil végétatif ont moins d'importance que ceux de l'appareil reproducteur. Mais il ne s'agit pas ici d'un polymorphisme vague ou désordonné, apparaissant au hasard. Ces formations mycéliennes présentent une grande fixité. Cela est si vrai que Grigorakis lui-même avait basé sur la présence constante de tortillons spiralés son genre Spiralia qu'il a depuis abandonné. Non seulement les organes pectinés sont passés sous silence mais les organes nodulaires ne sont mentionnés que pour être assimilés à de grosses chlamydospores ou à des fuseaux, ce qui laisserait croire que Grigorakis ne les a jamais vus.

Ce troisième principe repose donc sur une erreur d'appréciation des caractères morphologiques des dermatophytes.

4. - Un vice fondamental, à notre avis, pour une classification naturelle est la séparation profonde que G. et $\mathrm{G}$. veulent établir, à la suite de Vuillemin, entre les dermatophytes à cultures glabres et les autres, à cultures poussiéreuses ou duveteuses. De ce que les fuseaux soient absents et de ce que les aleuries soient rudimentaires, il n'en résulte pas forcément que ces champignons soient très 
éloignés de ceux qui possèdent en abondance des aleuries et des fuseaux. Si nous ne voyons pas apparaître ces organes, c'est peut-être, comme le dit excellemment Sabouraud, parce que nous ne savons pas préparer le milieu favorable. L'ensemble des autres caractères morphologiques et biologiques ne nous paraît pas permettre cette séparation. Quelles que soient ses affinités, le groupe des dermatophytes forme un tout bien homogène qu'il ne faut pas dissocier.

Quelques espèces importantes ne figurent pas dans la classification de G. et G. Où placer, par exemple, le Trichophyton pedis Ota, 1922, si fréquent en Mandchourie et le T. soudanense Joyeux, 1912, si répandu en Afrique occidentale; l'Achorion violaceum Bloch, 1911, qui donne des godets à l'état parasitaire, des thyrses et des fuseaux dans les cultures; le Microsporum equinum Bodin, 1896 ; le Trichophyton currii Chalmers et Marshall, 1914, qui forme de véritables périthèces, etc., etc. ? Ces regrettables lacunes laissent le lecteur dans l'incertitude.

Enfin G. et G. ont négligé d'indiquer les auteurs et de donner la synonymie de leurs genres et sous-genres, violant ainsi les articles 40 et 41 du Code de nomenclature, ce qui fait encore tomber leurs dénominations sous le coup du $\$ 5$ de l'article 51 .

C'est une tâche bien ingrate que d'établir des catégories. Dans la nature il n'y a que des individus et des faits, enchaînés les uns aux autres. On pèche donc toujours par quelque endroit lorsqu'on veut les séparer et les classer. Les travaux de Sabouraud, d'abord, puis ceux de Bodin et de quelques autres, nous ont fait connaître les faits en telle quantité et avec une telle perfection de détails que rien de réellement nouveau n'a été décrit dans la suite. On a donné des noms à des détails morphologiques ou précisé leur importance, mais les aleuries, par exemple, sont déjà parfaitement caractérisées par Sabouraud. Un grand nombre d'individus ont été étudiés et la plupart des espèces basées sur ces descriptions semiblent solidement établies et faciles à identifier. Les compressions proposées par G. et G. ne résistent pas à la critique, comme il ressort de l'argumentation de Sabouraud. On ne discute donc plus que sur les genres, c'est-à-dire sur la manière de répartir les espèces en catégories.

A ce sujet, un point me paraît avoir été méconnu par tout le monde. C'est la différence qui existe entre une clef de détermination et une classification naturelle. Prenez une Flore. Vous êtes conduit au nom spécifique par des tableaux synoptiques ou des clefs dichotomiques qui vous dirigent en vous faisant constater la présence ou l'absence de tels ou tels caractères morphologiques habilement choisis. Vous arrivez facilement ainsi à déterminer une plante, mais en marchant en aveugle et c'est tout au plus si vous êtes informé de 
la famille et du genre. Avec de l'attention et quelque habitude, vous arrivez sûrement au but. Mais essayez d'en faire autant avec une classification naturelle, où les classes, les ordres et les familles vous sont présentés dans leur enchaînement rationnel et où les caractères utilisés sont souvent, bien que fondamentaux, d'appréciation difficile. Si vous n'êtes pas rompu à la connaissance des plantes, vous n'arriverez pas à une détermination correcte, parce que vous vous heurterez tout d'abord à d'insurmontables difficultés morphologiques : vous n'aurez pas la graine pour compter les cotylédons ou il vous manquera le microscope pour voir s'il y a ou non des vaisseaux.

La même chose arrive pour les dermatophytes. Les trois anciens genres fondamentaux sont uniquement basés sur les caractères morphologiques du champignon à l'état parasitaire. Ils ne tiennent pas compte des caractères macroscopiques et microscopiques que présentent les mêmes champignons à l'état saprophytique, dans les cultures pures artificielles. Mais ces trois groupements sont excellents pour le triage et la détermination pratique des dermatophytes. Ils ne nous apprennent rien sur leurs affinités, mais ils nous renseignent immédiatement sur la nature de la lésion et sur le traitement approprié. Maintenant que les espèces sont bien connues, ils nous permettent même d'arriver à la détermination approchée de l'espèce, détermination qui sera confirmée par la culture. Mais il ne faut pas donner à ces genres plus de valeur que ne leur en ont attribué leurs créateurs : Gruby, Remak, Malmsten. Ce ne sont pas de véritables unités systématiques et c'est pourquoi ils n'ont pas été admis par les botanistes (Saccardo, Lindau, etc.). C'est aussi pourquoi G. et G., en voulant leur attribuer une valeur systématique, ont abouti à une si déplorable confusion.

La tentative au cours de laquelle j'ai collaboré avec Ota avait pour but de proposer une classification naturelle et de véritables unités systématiques, le tout basé sur les caractères macroscopiques et microscopiques des cultures, sans tenir compte des caracteres épharmoniques parasitaires. C'est pourquoi nous n'avors pas cru pouvoir conserver les genres Microsporum et Achorion, excellents pour la détermination des parasites, mais ne correspondant pas suffisamment à la morphologie microscopique des cultures. Nous avons gardé le genre Trichophyton, parce qu'il permettait la constitution d'un groupe homogène. G. et G. ont voulu mieux faire. Ils ont cru pouvoir fusionner la clef de détermination, donnée par les anciens genres, avec une classification naturelle, en partie calquée sur la nôtre. Notre impression, qui est aussi celle de notre éminent Maître R. Sabouraud, est qu'ils ont échoué. 